\title{
Aspectos fisiológicos da mandioca após a aplicação dos herbicidas fluazifop-p-butil e fomesafen ${ }^{1}$
}

\author{
Daniel Valadão Silva ${ }^{2}$, Hellen Martins da Silveira ${ }^{3}$ Evander Alves Ferreira ${ }^{4}$, Felipe Paolinelli de Carvalho ${ }^{5}$, \\ Manoel Delintro de Castro Neto ${ }^{6}$, Antonio Alberto da Silva $a^{7}$ Tocio Sediyama ${ }^{8}$
}

\section{RESUMO}

Acredita-se que alguns herbicidas possam influenciar a eficiência do uso da água, pelo efeito negativo direto sobre fotossíntese, transpiração e condutância estomática ou, indiretamente, pela redução da taxa metabólica da planta. Diante disso, objetivou-se, neste trabalho, avaliar a influência dos herbicidas fluazifop-p-butil e fomesafen, isolados e em mistura, sobre as características associadas ao uso da água, por cultivares de mandioca, visando a selecionar aqueles mais tolerantes aos referidos herbicidas. Realizou-se experimento, em casa de vegetação no delineamento de blocos casualizados, com quatro repetições. Adotou-se arranjo fatorial em esquema 5x7, constituído pela combinação de cinco cultivares de mandioca: Cacau-UFV, Platina, Coqueiro, Coimbra e IAC-12, com quatro doses da mistura comercial dos herbicidas fomesafen + fluazifop-p-butil $\left(0,5 ; 0,75 ; 1,0\right.$ e 1,5 $\left.\mathrm{L} \mathrm{ha}^{-1}\right)$, além da dose comercial recomendada de cada princípio ativo isolado e uma testemunha sem herbicida. As características avaliadas foram: condutância estomática $(g s)$, taxa de transpiração $(E)$ e eficiência do uso da água $(E U A)$. De maneira geral, a $E$, $g$ s e $E U A$ foram afetadas pela aplicação da mistura dos herbicidas. Os cultivares de mandioca apresentam diferentes níveis de sensibilidade ao fluazifop-p-butil + fomesafen, sendo que o cultivar Platina é o mais tolerante à aplicação da mistura. O fluazifop-p-butil mostrou-se seletivo para a cultura e o fomasafen causou efeitos negativos na fisiologia da mandioca.

Palavras-chave: transpiração, condutância estomática, controle químico, Manihot esculenta.

\section{ABSTRACT \\ Physiological responses of cassava to application of the herbicides fluazifop-p-butil and fomesafen}

Some herbicides can influence the water use efficiency by the direct negative effect on photosynthesis, transpiration and stomatal conductance, or indirect, by reduction of plant metabolic rate. Therefore, this study aimed to evaluate the influence of fluazifop-p-butil and fomesafen herbicides, alone and in mixture, on the characteristics associated with water use efficiency in cassava cultivars, seeking to select the most tolerant cultivar to these herbicides. The experiment was carried out in a greenhouse in a randomized block design with four repetitions. The factorial scheme $5 \times 7$ was

Recebido para publicação em 04/10/2012 e aprovado em 15/10/2013.

${ }^{1}$ Parte da dissertação de Mestrado da segunda autora defendida pelo Programa de Pós-Graduação em Fitotecnia pela Universidade Federal de Viçosa. Agência finaciadora: Capes e FAPEMIG.

${ }^{2}$ Engenheiro-Agrônomo, Mestre. Departamento de Fitotecnia, Universidade Federal de Viçosa, Campus Viçosa, Avenida Peter Henry Rolfs, s/n, 36.570-000, Viçosa, Minas Gerais, Brasil. danielvaladaos@yahoo.com.br (autor para correspondência).

${ }^{3}$ Engenheira-Agrônoma, Mestre. Departamento de Fitotecnia, Universidade Federal de Viçosa, Campus Viçosa, Avenida Peter Henry Rolfs, s/n, 36.570-000, Viçosa, Minas Gerais, Brasil. hellenufv@hotmail.com

${ }^{4}$ Engenheiro-Agrônomo, Doutor. Universidade Federal dos Vales do Jequitinhonha e Mucuri, Rodovia MGT 367, Km 583, 5000, Alto da Jacuba, Diamantina, Minas Gerais, Brasil. evanderalves@gmail.com

${ }^{5}$ Engenheiro-Agrônomo, Mestre. Departamento de Fitotecnia, Universidade Federal de Viçosa, Campus Viçosa, Avenida Peter Henry Rolfs, s/n, 36.570-000, Viçosa, Minas Gerais, Brasil. felipepaolinelli@yahoo.com.br

${ }^{6}$ Engenheiro-Agrônomo, Mestre. Departamento de Fitotecnia, Universidade Federal de Viçosa, Campus Viçosa, Avenida Peter Henry Rolfs, s/n, 36.570-000, Viçosa, Minas Gerais, Brasil.mdc.neto@hotmail.com

${ }^{7}$ Engenheiro-Agrônomo, Doutor. Departamento de Fitotecnia, Universidade Federal de Viçosa, Campus Viçosa, Avenida Peter Henry Rolfs, s/n, 36.570-000, Viçosa, Minas Gerais, Brasil. aasilva@ufv.br

${ }^{8}$ Engenheiro-Agrônomo, Doutor. Departamento de Fitotecnia, Universidade Federal de Viçosa, Campus Viçosa, Avenida Peter Henry Rolfs, s/n, 36.570-000, Viçosa, Minas Gerais, Brasil. t.sediyama@ufv.br 
adopted: the first factor was five cultivars: Cacau-UFV, Platina, Coqueiro, Coimbra e IAC-12; and the second factor was four doses of the commercial mixture of fomesafen + fluazifop-p-butil $\left(0.5 ; 0.75 ; 1.0\right.$ and $\left.1.5 \mathrm{~L} \mathrm{ha}^{-1}\right)$, in addition to the commercial dose recommended for each active ingredient isolated and a control without herbicide. The characteristics evaluated were: stomatal conductance $(g \mathrm{~s})$, transpiration rate $(E)$ and water use efficiency $(E U A)$. In general, $E, g \mathrm{~s}$ and $E U A$ were negatively affected by the application of the herbicide mixture. Cassava cultivars have different levels of sensitivity to fluazifop-p-butil + fomesafen, being the cultivar Platina more tolerant to application of the mixture. Fluazifop-p-butil showed selectivity to the cropand fomasafen caused negative effects on the physiology of cassava.

Key words: transpiration, stomatal conductance, herbicides, Manihot esculenta.

\section{INTRODUÇÃO}

Dentre as principais culturas do Brasil, a mandioca (Manihot esculenta Crantz) destaca-se pela sua importância social e econômica, sendo cultivada em 1,9 milhão de hectares e sua produção destina-se, principalmente, à fabricação de farinha, de fécula e ao consumo in natura (IBGE, 2013). A grande importância social dessa cultura deve-se à sua exploração em regiões de estações secas, onde ocorrem os mais baixos níveis de índice de desenvolvimento humano (IDH), do Brasil e do mundo (Cavalcanti, 2004).

A colheita da mandioca pode ocorrer entre 8 e 12 meses após o plantio, quando suas raízes são destinadas para o consumo in natura, e até dois anos após, quando a finalidade dessas for o processamento na indústria (Silva et al., 2012a). Em consequência deste longo período de cultivo, associado à cobertura parcial do solo promovido pela cultura, podem ocorrer, na área de plantio, várias infestações de plantas daninhas, resultando em perdas de produtividade superiores a 90\% (Moura, 2000; Johanns $\&$ Contiero, 2006).

Um dos grandes problemas para o controle eficiente das plantas daninhas, na cultura da mandioca, a baixo custo, é o pequeno número de herbicidas registrados para esta cultura. $\mathrm{O}$ fluazifop-p-butil é inibidor da enzima acetil-CoA carboxilase (ACCase), muito utilizado e eficiente para o controle de gramíneas, em culturas dicotiledôneas. Esta enzima, encontrada no estroma dos plastídeos, converte acetil coenzima-A (acetil-coA) a malonil coenzima-A, por adição de $\mathrm{CO}_{2}\left(\mathrm{HCO}_{3}\right)$ à acetil coA, no primeiro passo do processo de biossíntese dos ácidos graxos. A inibição da síntese de ácidos graxos bloqueia a produção de fosfolipídeos (Burke et al., 2006), usados na construção de novas membranas necessárias para o crescimento celular.

O fomesafen pertence ao grupo dos inibidores da enzima protoporfirinogênio oxidase (PROTOX). É utilizado, nas culturas do feijão e da soja, para o controle de espécies de folhas largas anuais, entre elas Acanthospermum australe, Amaranthus hybridus, Euphorbia heterophylla, Bidens pilosa, Ipomoea grandifolia, além de outras (Silva et al., 2007). A mistura pronta desses herbicidas é o principal produto comercial utilizado na cultura do feijoeiro, sendo muito eficiente para o controle de plantas daninhas gramíneas e dicotiledôneas (Fontes et al., 2001).

Em diversos estudos com culturas, tem-se observado tolerância diferencial, de genótipos de uma mesma espécie, a herbicidas. Para cana-de-açúcar, Ferreira et al. (2005) e Galon et al. (2010) relataram diferença na tolerância, de genótipos, aos herbicidas ametryn e trifloxysulfuronsodium. Efeitos semelhantes foram observados por Rocha et al. (2010), para pinhão-manso, quando tratado com herbicidas aplicados em pré-emergência. Normalmente, efeitos diferenciais de herbicidas entre cultivares são observados quando se avalia não apenas a produtividade, mas, também, os efeitos sobre os fatores de crescimento das plantas (Negrisoli et al., 2004; Galon et al., 2009).

Acredita-se que herbicidas possam influenciar a eficiência do uso da água, pelo efeito negativo direto sobre a fotossíntese, ou, indiretamente, pela redução da taxa metabólica da planta. Contudo, especula-se a existência de diferenças marcantes, entre os materiais genéticos de uma mesma espécie, que podem ser avaliadas quanto a diferenças na condutância estomática, taxa transpiratória, temperatura da folha e acúmulo de matéria seca, quando eles são tratados com herbicidas (Galon et al., 2010). Dessa forma, a associação genótipo / herbicida pode determinar aqueles que sobressaem sob estresse hídrico, quando sob aplicação do produto e, assim, garantir melhor produção da cultura.

Neste trabalho, objetivou-se avaliar a influência dos herbicidas fluazifop-p-butil e fomesafen, isolados ou em mistura, sobre as características associadas ao uso da água, em cultivares de mandioca, visando a selecionar aqueles mais tolerantes à ação desses herbicidas.

\section{MATERIAL E MÉTODOS}

O experimento foi conduzido em casa de vegetação, com condições controladas de temperatura e umidade. As plantas foram cultivadas em vasos de $12 \mathrm{~L}$, preenchidos 
com um Latossolo Vermelho-Amarelo distrófico típico, textura argilosa (56\% argila, $6 \%$ silte e $38 \%$ areia). De acordo com a análise química, o solo apresentava $\mathrm{pH}$ (água) de 5,4; teor de matéria orgânica de 1,8 dag kg-1; $\mathrm{P}, \mathrm{K}, \mathrm{Ca}$, $\mathrm{Mg}, \mathrm{Al}, \mathrm{H}+\mathrm{Al}$ e $\mathrm{CTC}_{\text {efetiva }}$ de 1,$4 ; 10 ; 0,5 ; 0,2 ; 0,4 ; 4,4$ e 1,7 $\mathrm{cmol}_{\mathrm{c}} \mathrm{dm}^{-3}$, respectivamente. Para adequação do substrato, quanto à nutrição, foram aplicados o equivalente a $300 \mathrm{~kg} \mathrm{ha}^{-1}$ de calcário dolomítico, $220 \mathrm{~kg} \mathrm{ha}^{-1} \mathrm{de}$ super fosfato simples e $40 \mathrm{~kg} \mathrm{ha}^{-1}$ de cloreto de potássio. A adubação nitrogenada foi realizada, em cobertura, aos 30 dias após a emergência da cultura, na dose de $40 \mathrm{~kg}$ ha ${ }^{1}$ de ureia, previamente dissolvida em água. As irrigações foram realizadas conforme a necessidade da cultura.

O delineamento experimental utilizado foi o de blocos casualizados, em arranjo fatorial 5x7, com quatro repetições, com uma planta por vaso sendo a parcela. Os tratamentos foram constituídos pela combinação de cinco cultivares de mandioca (Cacau-UFV, Platina, Coqueiro, Coimbra e IAC-12), com cinco doses $(0,00 ; 100+125$; $150+187,5 ; 200+250$ e $300+375 \mathrm{~g} \mathrm{ha}^{-1}$, equivalente a 0,00 ; 0,$50 ; 0,75 ; 1,00$ e $1,50 \mathrm{~L}$ da mistura comercial) de fomesafen + fluazifop-p-butil, além da dose de cada princípio ativo isolado (200 e $250 \mathrm{~g} \mathrm{ha}^{-1}$ de fluazifop-p-butil e fomesafen, respectivamente, equivalente a $1 \mathrm{~L} \mathrm{ha}^{-1}$ ).

Efetuou-se o plantio das manivas, no mês de março de 2011, e a emergência ocorreu cinco dias após. A aplicação dos herbicidas foi realizada aos 30 dias após o plantio, com pulverizador costal, pressurizado a $\mathrm{CO}_{2}$, com pressão constante de $200 \mathrm{kPa}$, equipado com uma barra de dois bicos de indução de ar, trabalhando a altura de $50 \mathrm{~cm}$ do alvo, com velocidade de $1 \mathrm{~m} \mathrm{~s}^{-1}$, atingindo faixa aplicada de $50 \mathrm{~cm}$ de largura e propiciando volume de calda de $150 \mathrm{~L} \mathrm{ha}^{-1}$.

Decorridos 30 dias da aplicação dos herbicidas, foram realizadas as avaliações, no terço médio da primeira folha completamente expandida. Foi utilizado analisador de gases no infravermelho (IRGA), sendo avaliada a condutância estomática de vapores de água $\left(\mathrm{gs}-\mathrm{mol} \mathrm{m}^{-1} \mathrm{~s}^{-1}\right)$ e a taxa de transpiração $\left(E-\mathrm{mol} \mathrm{H}_{2} \mathrm{O} \mathrm{m}^{-2} \mathrm{~s}^{-1}\right)$. Calculou-se, ainda, a eficiência do uso da água $\left(E U A-\mathrm{mol} \mathrm{CO}_{2} \mathrm{~mol}\right.$ $\mathrm{H}_{2} \mathrm{O}^{-1}$ ), a partir dos valores de quantidade de $\mathrm{CO}_{2}$ fixado pela fotossíntese e quantidade de água transpirada.

Todos os dados foram submetidos à análise de variância pelo teste F. Para o fator qualitativo as médias foram comparadas utilizando-se o teste de Tukey e para comparar a testemunha com as demais doses optou-se pelo teste de Dunnett adotando-se o nível de 5\% de probabilidade.

\section{RESULTADOS E DISCUSSÃO}

Constatou-se interação significativa entre os fatores dose de herbicidas e cultivares de mandioca, para todas as variáveis avaliadas, sendo, portanto, desmembrada para estudo. Para a taxa transpiratória $(E)$, a ausência dos herbicidas (dose 0), o cultivar Platina apresentou os valores mais elevados sem, entretanto, diferir de Coqueiro e de Cacau-UFV (Tabela 1). No momento da avaliação, as plantas encontravam-se em pleno desenvolvimento do sistema radicular e da parte aérea (Peressin \& Carvalho, 2002); assim, espera-se que cultivares com maiores $E$ apresentem maiores produções de matéria seca, como foi observado por Silva et al. (2011) para os mesmos cultivares.

A mistura de fluazifop-p-butil + fomesafen não afetou a $E$ dos cultivares Coqueiro e Cacau-UFV, mesmo nas maiores doses (Tabela 1). No entanto, Coimbra e IAC-12 tiveram aumento de sua $E$ em relação a testemunha, a partir das doses 1 e 0,5, respectivamente. Os herbicidas aplicados na forma isolada pouco afetaram a variável, com exceção do caso de Coqueiro, para o qual ela apresentou redução pela aplicação do fluazifop-p-butil.

As doses 0,75, 1 e 1,5 da mistura (fluazifop-p-butil + fomesafen) provocaram aumento da condutância estomática ( $g s$ ) dos cultivares Coqueiro e Cacau-UFV (Tabela 2). O mesmo foi constatado para a IAC-12 a partir da dose $0,5 \mathrm{e}$ para a Coimbra nas doses de 1 e 1,5. O cultivar Cacau-UFV apresentou os maiores valores de gs, quando tratado com a maior dose da mistura, sendo que, sem a presença dela (dose 0), observaram-se os menores valores da variável, ocorrendo o mesmo com Coimbra. Os herbicidas aplicados isoladamente não afetaram a variável.

O fluazifop-p-butil é um inibidor da enzima acetil-CoA carboxilase (ACCase), que catalisa a primeira etapa, das sete, na rota biossintética de ácidos graxos, convertendo o Acetil Coenzima A (Acetil-CoA) em Malonil Coenzima A (Malonil-CoA), pela adição de uma molécula de CO (Silva \& Silva, 2007). Os inibidores dessa enzima bloqueiam a incorporação do $\mathrm{CO}_{2}$ impedindo a síntese de lipídios em plantas susceptíveis. Parte da tolerância da mandioca as menores doses da mistura está relacionada com a insensibilidade enzimática da mandioca ao fluazifop-p-butil e com a metabolização do fomesafen, que chega em menor quantidade ao sítio de ação, provocando, desta forma, incremento dos valores destas variáveis. Esses resultados concordam com os observados por Silva et al. (2012b), que relataram que o herbicida fluazifop-p-butil mostrouse promissor para aplicação em área total, uma vez que foi altamente seletivo para a cultura da mandioca.

Já o fomesafen atua na planta através da inibição da enzima PROTOX, localizada nos cloroplastos, reduzindo a síntese das clorofilas. Com isso, ocorrem o acúmulo de protoporfirinogênio IX e sua saída para o citoplasma, onde é oxidado, formando a protoporfirina IX (Jacobs et al., 1991), que, por sua vez, interage com oxigênio e luz, formando oxigênio "singlet", uma espécie reativa de oxigênio (ROS), que desencadeia processos oxidativos, como a peroxidação de lipídios das membranas (Jacobs et al., 
1991; Hess, 2000; Tripathy et al., 2007). Desta forma, o fomesafen tem ação nos cloroplastos, afetando, diretamente, a fotossíntese, e, indiretamente, a transpiração e condutância estomática, enquanto o fluazifop-p-butil age no citosol e tem ação indireta na fotossíntese, transpiração e condutância. Segundo Silva et al. (2011) a aplicação da mistura e do fomesafen isolado provocou sintomas de intoxicação como retorcimento e necroses do tecido das folhas, e esses sintomas eram potencializados na medida em que se aumentava a dose. Todavia, os mesmos autores relataram que os sintomas eram reduzidos na medida em que surgiam novas folhas na planta, sendo esse o provável motivo dos menores efeitos do fomesafen observados para a $E$ e $g$ s, visto que a avaliação ocorreu aos 30 dias após a aplicação dos herbicidas.

Avaliando-se os cultivares na ausência de herbicidas, verifica-se que a IAC-12 e o Coqueiro mostraram-se maiores eficiência do uso da água eficientes (EUA) do que a Platina e Cacau-UFV (EUA) (Tabela 3). No entanto, na maior dose da mistura $(1,5)$ os cultivares Coqueiro e Cacau-UFV apresentaram maior EUA do que dos demais cultivares. Admitindo-se as possíveis diferenças em função do centro de domesticação e da amplitude do território brasileiro, a obtenção dos cultivares Coqueiro e IAC-
12 pode ser diferente da de outras, como Cacau-UFV, sendo que os dois primeiros possuem boa adaptação a ambientes de semiáridos (Lopes et al., 2010) e, o último, a ambientes com maiores regimes de chuva.

Com a aplicação dos herbicidas, o IAC-12 apresentou redução da EUA, em relação a testemunha, na dose de 1,5 L/ha na mistura dos produtos e para o fomesafen isolado (Tabela 3). Apesar de não ser afetado pela mistura dos herbicidas, o cultivar Coqueiro teve sua EUA reduzida, quando tratado com o fomesafen. Admitindo-se a menor tolerância ao fomesafem, observada nas plantas que receberam cada produto isoladamente, é provável que o efeito da mistura contenha menor participação do fluazifop-p-butil. Para os demais cultivares, não foi observado o mesmo comportamento, com exceção de Platina, que apresentou maior EUA para a dose $0,75 \mathrm{~L} \mathrm{ha}^{-1}$. Aspiazu et al. (2010), trabalhando com a cultura da mandioca em condição de competição, verificaram que a cultura apresentou maior EUA, comparada com a das plantas daninhas testadas. A EUA é influenciada por diversos fatores ambientais, como a temperatura da folha, a velocidade do vento e a umidade relativa. Em condições ideais, as plantas de mandioca são mais eficientes em uso da água do que diversas outras espécies cultivadas (El-Sharkawy, 2003).

Tabela 1. Taxa transpiratória $\left(\mathrm{E}-\mu \mathrm{mol} \mathrm{H}_{2} \mathrm{O} \mathrm{m}^{-2} \mathrm{~s}^{-1}\right)$ dos cultivares de mandioca tratados com doses crescentes de fluazifop-p-butil + fomesafen e isoladamente

\begin{tabular}{|c|c|c|c|c|c|c|c|}
\hline \multirow{3}{*}{ Cultivares } & \multicolumn{5}{|c|}{ Fluazifop-p-butil + Fomesafen } & \multirow[t]{2}{*}{ Fluazifop-p-butil } & \multirow[t]{2}{*}{ Fomesafen } \\
\hline & \multicolumn{5}{|c|}{ Lha $^{-1}$} & & \\
\hline & $\mathbf{0}$ & 0,5 & 0,75 & 1 & 1,5 & 1 & \\
\hline Coimbra & $2,8 \mathrm{~b}$ & $3,5 \mathrm{a}$ & $3,5 \mathrm{~b}$ & $4,1 \mathrm{a}^{*}$ & $4,2 *$ & $2,6 \mathrm{ab}$ & $2,7 \mathrm{~b}$ \\
\hline Platina & $4,9 \mathrm{a}$ & $3,7 \mathrm{a}$ & $3,2 b^{*}$ & $2,7 b^{*}$ & $3,8 \mathrm{a}$ & $4,2 \mathrm{a}$ & $3,4 \mathrm{a}$ \\
\hline IAC-12 & $3,0 \mathrm{~b}$ & $4,2 a^{*}$ & $4,9 a^{*}$ & $4,3 \mathrm{a}^{*}$ & $4,6 a^{*}$ & $3,1 \mathrm{ab}$ & $3,6 \mathrm{a}$ \\
\hline Coqueiro & $3,5 \mathrm{ab}$ & $3,9 \mathrm{a}$ & $4,1 \mathrm{ab}$ & $4,2 \mathrm{a}$ & $4,3 \mathrm{a}$ & $2,9 a b$ & $4,0 \mathrm{a}$ \\
\hline Cacau-UFV & $3,5 \mathrm{ab}$ & $4,1 \mathrm{a}$ & $3,6 \mathrm{~b}$ & $4,2 \mathrm{a}$ & $4,3 \mathrm{a}$ & $2,1 \mathrm{~b}^{*}$ & $3,5 \mathrm{a}$ \\
\hline
\end{tabular}

Médias seguidas pela mesma letra minúscula na coluna não diferem entre si pelo teste de Tukey a 5\% de probabilidade. Médias seguidas por * na linha diferem da testemunha (dose 0) ao nível de $5 \%$ de probabilidade pelo teste de Dunnett.

Tabela 2. Condutância estomática $\left(g \mathrm{~s}-\mu \mathrm{mol} \mathrm{m} \mathrm{m}^{-1} \mathrm{~s}^{-1}\right)$ dos cultivares de mandioca tratados com doses crescentes de fluazifop-p-butil + fomesafen e isoladamente

\begin{tabular}{|c|c|c|c|c|c|c|c|}
\hline \multirow{3}{*}{ Cultivares } & \multicolumn{5}{|c|}{ Fluazifop-p-butil + Fomesafen } & \multirow[t]{2}{*}{ Fluazifop-p-butil } & \multirow[t]{2}{*}{ Fomesafen } \\
\hline & \multicolumn{5}{|c|}{ L ha $^{-1}$} & & \\
\hline & $\mathbf{0}$ & 0,5 & $\mathbf{0 , 7 5}$ & 1 & 1,5 & 1 & \\
\hline Coimbra & $0,23 \mathrm{~b}$ & $0,48 \mathrm{ab}$ & $0,47 \mathrm{~b}$ & $0,65 \mathrm{ab}^{*}$ & $0,75 a b^{*}$ & $0,21 \mathrm{~b}$ & $0,21 \mathrm{~b}$ \\
\hline Platina & $0,42 \mathrm{a}$ & $0,33 \mathrm{~b}$ & $0,34 \mathrm{~b}$ & $0,30 \mathrm{~b}$ & $0,52 \mathrm{~b}$ & $0,50 \mathrm{a}$ & $0,45 \mathrm{a}$ \\
\hline IAC-12 & $0,45 \mathrm{a}$ & $0,86 \mathrm{a}^{*}$ & $0,84 \mathrm{a}^{*}$ & $0,88 \mathrm{~b}^{*}$ & $0,70 \mathrm{ab}^{*}$ & $0,46 \mathrm{a}$ & $0,58 \mathrm{a}$ \\
\hline Coqueiro & $0,43 \mathrm{a}$ & $0,49 \mathrm{ab}$ & $0,68 \mathrm{ab}^{*}$ & $0,97 b^{*}$ & $0,76 a b^{*}$ & $0,23 \mathrm{~b}$ & $0,43 \mathrm{a}$ \\
\hline Cacau-UFV & $0,31 \mathrm{~b}$ & $0,53 \mathrm{ab}$ & $0,68 \mathrm{~b}^{*}$ & $0,77 \mathrm{ab}^{*}$ & $1,20 \mathrm{a}^{*}$ & $0,19 \mathrm{~b}$ & $0,42 \mathrm{a}$ \\
\hline
\end{tabular}

CV(\%) 48,31

Médias seguidas pela mesma letra minúscula na coluna não diferem entre si pelo teste de Tukey a 5\% de probabilidade. Médias seguidas por

* na linha diferem da testemunha (dose 0) ao nível de $5 \%$ de probabilidade pelo teste de Dunnett. 
Tabela 3. Eficiência do uso da água (EUA- $\mu \mathrm{mol} \mathrm{CO} \mathrm{Col} \mathrm{H}_{2} \mathrm{O}^{-1}$ ) dos cultivares de mandioca tratados com doses crescentes de fluazifop-p-butil + fomesafen e isoladamente

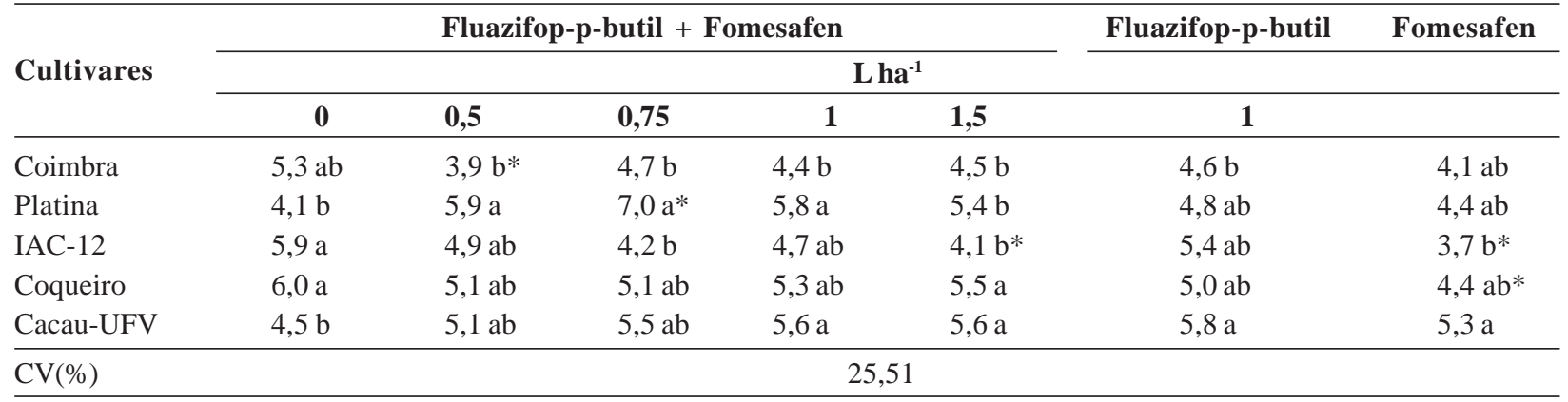

Médias seguidas pela mesma letra minúscula na coluna não diferem entre si pelo teste de Tukey a 5\% de probabilidade. Médias seguidas por

* na linha diferem da testemunha (dose 0 ) ao nível de $5 \%$ de probabilidade pelo teste de Dunnett.

De maneira geral, a mistura dos herbicidas mostrouse tóxica para a mandioca. Possivelmente, essa toxicidade é agravada pelos aditivos adicionados aos ingredientes ativos para a formação do produto comercial, pois o efeito negativo da mistura foi maior que o observado na aplicação do fluazifop-p-butil e do fomesafen, nas aplicações isoladas. Esses resultados indicam a possibilidade de aplicação, em pós-emergência, do fluazifop-p-butil, em área total. Para o fomesafen, pesquisas de campo são necessárias, para avaliar se a intoxicação visual (Silva et al., 2011, 2012b) e a fisiológica, observadas neste trabalho, causam efeitos negativos na produtividade da cultura.

\section{CONCLUSÕES}

Os cultivares de mandioca apresentam diferentes níveis de tolerância ao fluazifop-p-butil + fomesafen, sendo que Platina é o mais tolerante à aplicação da mistura.

O fluazifop-p-butil mostrou-se seletivo quanto à cultura, e o fomasafen causou efeitos negativos na fisiologia da cultura.

\section{AGRADECIMENTOS}

Ao Conselho Nacional de Desenvolvimento Científico e Tecnológico (CNPq), à Fundação de Amparo à Pesquisa do Estado de Minas Gerais (FAPEMIG) e à Coordenação de Aperfeiçoamento de Pessoal de Nível Superior (CAPES), pelo financiamento e suporte para a execução do trabalho.

\section{REFERÊNCIAS}

Aspiazú I, Sediyama T, Ribeiro Jr JI, Silva AA, Concenço G, Ferreira EA, Galon L, Silva AF, Borges ET, Araujo WF (2010) Photosynthetic activity of cassava plants under weed competition. Planta Daninha, 28:963-968.

Brodribb TJ \& Hill RS (2000) Increases in water potential gradient reduce xylem conductivity in whole plants. Evidence from a lowpressure conductivity method. Plant Physiology, 12:1021-1028.
Burke IC, Thomas WE, Burton JD, Spears JF, Wilcut JW (2006) A seedling assay to screen aryloxyphenoxypropionic acid and cyclohexanedione resistance in johnsongrass (Sorghum halepense). Weed Technology, 20:950-955.

Calvancanti J (2004) Perspectivas da mandioca na região semiárida do Nordeste. Disponível em: <http://www.embrapa.br/imprensa/artigos/2002/artigo.2004-12-07.26079 18288/>. Acessado em: 10 de fevereiro de 2012.

Ferreira EA, Santos JB, Silva AA, Ventrella MC, Barbosa MHP, Procópio SO, Rebello VPA (2005) Sensibilidade de cultivares de cana-de-açúcar à mistura trifloxysulfuron-sodium + ametryn. Planta Daninha, 23:93-99.

Fontes JRA, Araujo GAA, Silva AA, Cardoso AA (2001). Efeitos de herbicidas no controle de plantas daninhas na cultura do feijão-mungo-verde (Vigna radiata (L.) Wilczek). Ciência e Agrotecnologia, 25:1087-1096.

Galon L, Ferreira FA, Ferreira EA, Silva AA, Silva AF, Aspiazú I, Concenço G, Fialho CMT, Santos EA, Tironi SP, Barbosa MHP (2009) Seletividade de herbicidas a genótipos de cana-deaçúcar. Planta Daninha, 27:1083-1093.

Galon L, Concenço G, Ferreira EA, Aspiazú I, Silva AF, Ferreira FA, Silva AA, Tironi SP, Freitas MAM, Soares ER (2010) Eficiência de uso da água em genótipos de cana-de-açúcar submetidos à aplicação de herbicidas. Planta Daninha, 28:777-784.

Hess FD (2000) Light-dependent herbicides: an overview. Weed Science, 48:160-170.

IBGE Instituto Brasileiro de Geografia e Estatística. Sétima previsão da safra 2012/2013. Disponível em: <http:// www1.ibge.gov.br/home/presidencia/noticias /noticia_ visualiza.php?id_noticia=1798\&id_pagina=1>. Acessado em: 10 de junho de 2013.

Jacobs JM, Jacobs NJ, Sherman TD, Duke SO (1991) Effects of diphenyl ether herbicides on oxidation of protoporphyrinogen to protoporfphyrin in organellar and plasma membrane enriched fractions of barley. Plant Physiology, 97:197-203.

Johanns O \& Contierio R (2006) Efeitos de diferentes períodos de controle e convivência de plantas daninhas com a cultura da mandioca. Revista Ciência Agronômica, 37:326-331.

Lopes AC, Viana AES, Matsomoto SN, Cardoso Jr NS, José ARS (2010) Complementação da irrigação e épocas de colheita de mandioca cv. coqueiro no planalto de conquista, BA. Ciência e Agrotecnologia, 34:579-587.

Peressin VA, Carvalho JEB (2002) Manejo integrado de plantas daninhas em mandioca. In: Marney Pascoli Cereda. (Org.). Cultura de Tuberosas Amiláceas Latino Americanas. São Paulo: Fundação Cargill, 2:302-349. 
Rocha PRR, Silva AF, Faria AT, Galon L, Ferreira EA, Felipe RS, Silva AA, Dias LAS (2010) Seletividade de herbicidas pré-emergentes ao pinhão-manso (Jatropha curcas). Planta Daninha, 28:801-806.

Silva AA \& Silva JF (2007) Tópicos em manejo de plantas daninhas. Silva AA \& Silva JF (Eds.). Viçosa, Universidade Federal de Viçosa. p.17-62

Silva DV, Santos JB, Ferreira EA, França AC, Silva AA, Sediyama T (2012a) Manejo de plantas daninhas na cultura da mandioca. Planta Daninha, 30:901-910.
Silva DV, Santos JB, Carvalho FP, Ferreira EA, França AC, Fernandes JSC, Gandini EMM, Cunha VC. (2012b). Seletividade de herbicidas pós-emergentes na cultura da mandioca. Planta Daninha, 30:835-841.

Silva DV, Santos JB, Silveira HM, Carvalho FP, Castro Neto MD, Ferreira EA, Silva AA, Cecon PR (2011) Tolerância de cultivares de mandioca aos herbicidas fomesafen e fluazifop-p-butil. Revista Brasileira de Herbicidas, 10:219-231.

Tripathy BC, Mohapatra A \& Gupta I (2007) Impairment of the photosynthetic apparatus by oxidase stress induced by photosensitization reaction of protoporphyrin IX. Biochimica et Biophysica Acta, 6:860-868. 